\title{
Grammatical category as mediator ${ }^{1}$
} ZENON W. PYLYSHYN and ANDREW FELDMAR,
University of Western Ontario, London, Canada

English words which are ambiguous with respect to their noun-verb part of speech were paired with nonsense words rendered unambiguous with respect to part of speech by appending suitable noun or verb endings. After PA learning trials a posttest revealed that the homograph with the same part of speech as that of its nonsense word associate tended to be more frequently elicited. This result was interpreted in terms of the operation of grammatical category as mediator. The utility of such a construct for theories of sentence generation was discussed.

A number of models of sentence generation have contained the implicit assumption that grammatical form class is an attribute which can be independently manipulated by psychological mechanisms-in other words that it has psychological properties akin to those displayed by a variety of mediational processes. Osgood (1963), for example, proposes that sentence generation involves the selection of words from a "Word Form Pool" through a series of "Semantic Key Sorts." One of these sorts is a grammatical sort which selects items of the appropriate grammatical category in the same manner as words are selected which meet the desired associative, affective, and denotative requirements for the intended sentence.

A model proposed by Yngve (1964) postulates abstract mediators closely related to grammatical category. Yngve refers to these constructs as "commitments" on the part of the speaker to finish his sentence grammatically. At each point in the speaking of a sentence the speaker incurs a commitment to utter certain grammatical constructions (e.g., a noun phrase, a noun, a verb, etc.), otherwise the sentence would not end up being grammatical.

Garrett \& Fodor (1968) also argue that a mediational account of language behavior would at the very least require such mediating constructs as "noun phrase," "verb phrase," "adjective," and the like.

The present experiment was designed to determine whether grammatical category could serve as an implicit mediator in a paired-associates task. English words which are highly ambiguous with respect to part of speech (as well as meaning) were paired with nonsense words that had been rendered unambiguous with respect to part of speech by affixing suitable verb or noun endings. After an initial learning phase the ambiguous words were tested in a sentence-construction task to determine the relative frequency of use of their noun and verb homographs. The assumption was that if part of speech had served as mediator in the learning phase, the homograph with the same part of speech as that of the nonsense word (and hence of the mediator) would be most readily elicited in the posttest.

\section{METHOD}

The eight ambiguous words (A) used in this study were all of frequency higher than 100 per million in the ThorndikeLorge count. They were selected for maximum noun/verb ambiguity from a pilot study using 36 Ss. A more comprehensive list of homograph norms is now available (Pylyshyn \& Feldmar, 1968).

In order to control for prior associations of the ambiguous with the unambiguous members of the pairs the latter were constructed as follows. Eight nonsense syllables of $O \%$ associative value (Glaze, 1928) were chosen: CIJ, QUG, DAX, LAJ, YOP, ZIQ, FEH, and XAD. Each of these was expanded into a nonsense word by appending either a noun $(N)$ or a verb
(V) ending to it. There were four noun endings (-INESS, -ILITY, -ISM, -DOM) and four verb endings (-IVATE, -ILIZE, -EFY, -IFY).

Four paired-associates lists were constructed from these words as follows. In the first list $(\mathrm{A}-\mathrm{N})$ the ambiguous words served as stimuli. These were randomly paired with noun-like nonsense words constructed by appending each of the four noun endings to two CVC trigrams. List $2(\mathrm{~A}-\mathrm{V})$ was the same except that verb endings were appended to the trigrams before pairing them randomly with different ambiguous words. Lists 3 (N-A) and 4 (V-A) were constructed using exactly the same procedure as for Lists 1 and 2 except that the role of stimulus and response members was reversed; unambiguous nonsense words served as stimuli while ambiguous words were now the response members.

The word pairs and the stimulus words alone were each photographed on $35 \mathrm{~mm}$ high contrast film and the four lists were presented to different groups of Ss using a projector. Ss were drawn from the introductory psychology classes. List A-N was presented to $21 \mathrm{Ss}$, List A-V to $23 \mathrm{Ss}$, List N-A to 25 Ss, and List V-A to 28 Ss. Forty-seven of the 97 Ss were males. Each group received 10 trials. A trial consisted of a presentation of all eight pairs (each time in a different random order) followed by a presentation of the individual stimulus words alone. Ss wrote their responses on sheets of paper containing eight numbered lines. Timing was as follows: Pairs were exposed for $3 \mathrm{sec}$ with a 1-sec pause between pairs. Stimulus words alone were exposed for $10 \mathrm{sec}$ intervals to allow sufficient time to record the response. A 5-sec delay separated presentation of lists. Two practice trials on a list of four paired associates were given to each group. The material in these trials consisted of unambiguous English words and grammatically neutral nonsense words in the same order as in the main experiment for that group.

To remove a possible source of ambiguity as to the part of speech of nonsense words in the experiment (e.g., ZIQIVATE might be misconstrued as a noun or adjective if pronounced zik'ivet), $E$ read aloud all the nonsense words during presentation of pairs. The instructions to the Ss (identical for all groups) also suggested that they should pronounce all the words to themselves while they were being presented.

After the learning phase Ss were given a list of the stimulus words and asked to write the response word and then to write a short phrase or sentence using each of the English words. These were subsequently categorized as to part of speech (noun or verb) independently by two judges. The judges agreed on 740 of the 762 phrases and after discussion agreed on a consistent procedure for categorizing the remaining ones.

\section{RESULTS}

The Ss were randomly eliminated from each of the four groups so that 20 Ss were left per group. A 2 by 2 analysis of variance was performed on the number of ambiguous words used as nouns. This score thus had a range of $0-8$. The two factors in the analysis were (a) whether the ambiguous word had been paired with a noun-like or verb-like nonsense word, and (b) whether it had served as stimulus or response in the learning trials.

The results showed that ambiguous words were used significantly more often in the same part of speech as that of the nonsense words with which they had been paired $(F=11.5 ; \mathrm{df}=1,76 ; \mathrm{p}<.01)$. Whether the ambiguous word was used as stimulus or response did not in itself lead to a main effect, but the interaction of this factor with part of speech usage was significant $(F=5.1 ; \mathrm{df}=1,76 ; \mathrm{p}<.05)$. The nature of this interaction is evident from the means given in Table 1. The experimental manipulation of the part of speech 
Table 1

Mean Number of Ambiguous Words (out of 8) Used as Nouns in the Post-Test for Each of the Experimental Conditions

\begin{tabular}{lccc} 
& \multicolumn{2}{c}{ Ambiguous word used as: } \\
\cline { 3 - 4 } & Stimulus & Response \\
\hline \multirow{2}{*}{ paired with } & $\begin{array}{l}\text { nonsense "noun" } \\
\text { nonsense "verb" }\end{array}$ & 5.8 & 6.7 \\
\hline
\end{tabular}

of the ambiguous word most often used in the posttest is most evident when the ambiguous word was used as the response in the PA task. Figure 1 shows the frequency with which each of the eight ambiguous words were used as nouns after being paired with noun-like and verb-like nonsense words.

\section{DISCUSSION}

The results clearly confirm the prediction that the grammatical usage of an ambiguous word can be influenced by pairing it with a nonsense word of a given part of speech.

The finding that this effect is lessened when the nonsense word is used as stimulus in the learning phase is best explained by considering the well known difficulty of determining what was in fact the "functional stimulus" in the learning. Underwood (1963) has shown that with college students, and especially when the stimulus is low on meaningfulness, there is a strong tendency for only a small part of the overall stimulus to be selected as the "functional" stimulus for the learning. In fact the minimally necessary differentiating component is most likely to be used. In the present experiment this might well have been the first letter of the nonsense word. Thus little attention might have been paid to those features of the nonsense word which establish its part of speech.

The main result of this experiment also reinforces the original belief that grammatical category (at least the noun vs verb distinction) may play a mediating role in verbal behavior. The argument for this may be illustrated by drawing a parallel with an example which involves logical category as mediator. Suppose two groups of Ss were each required to learn a PA list. The first list contained the pair "cannon-tank" and the other list contained the pair "bottle-tank". The word "tank" here is ambiguous. It can mean either a war vehicle $\left(\operatorname{tank}_{1}\right)$ or container $\left(\tan k_{2}\right)$. One might speculate that "cannon-tank $k_{1}$ "

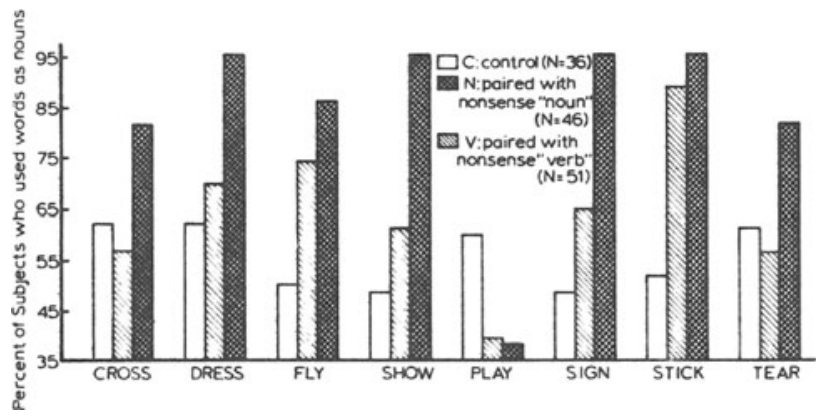

Fig. 1. Frequency of use of noun homograph for each ambiguous word after pairing with "noun" and "verb" nonsense words. is simply mediated by the concept "armament" while "bottle-tank $k_{2}$ " is simply mediated by the concept "container." The remaining combinations cannot be as simply mediated by obvious categorical mediators. Thus one might expect that those learning List 1 might be learning the response tank $k_{1}$ while those learning List 2 might be learning the response tank $_{2}$. This might subsequently be tested by asking Ss to write a brief sentence containing the word "tank." This task would be expected to reveal $\operatorname{tank}_{1}$ or tank $\mathrm{k}_{2}$ depending on which had been recently "primed" by the learning task. The present experiment was very similar to this except that grammatical category was the potential mediator rather than logical category as in the above illustration. Also an attempt was made to control for other possible mediators by using nonsense words as associates. Thus with grammatical category the only systematic property in common between all stimulus and response items in each PA list, grammatical category is then the common potential mediator. The results of the posttest showed that the homograph with the same part of speech as that of its associate was most readily available, thus suggesting that it had been most recently "primed." This effect pf "priming" or of a set for an abstract property of a word which was provided by a nonsense word associate may be attributed to the pairing of stimulus and response members through the mediation of grammatical category.

Although this experiment was confined to individual words there is no obvious reason why "nounness" or "verbness" could not equally be a psychological attribute of the function performed by phrases. In other words, these results would suggest that it might be useful to consider the abstract symbols of generative grammars (i.e., NP, VP, N, V, D, etc.) as having psychological equivalents in the form of mediators. Note that this is not something that can be assumed a priori since there are many features of an abstract theory of language competence which need not find a parallel in a psychological mechanism. This is why it is important to establish empirically the psychological reality of such concepts as grammatical category.

\section{REFERENCES}

GARRETT, M., \& FODOR, J. Psychological theories and linguistic constructs. In T. R. Dixon and D. L. Horton (Eds.), Verbal behavior and general behavior. New Jersey: Prentice-Hall, 1968.

GLAZE, J. A. The association value of nonsense syllables. Journal of Genetic Psychology, 1928, 35, 255-267.

OSGOOD, C. On understanding and creating sentences. American Psychologist, 1963, 18, 735-751.

PYLYSHYN, Z. W., \& FELDMAR, A. Usage norms for noun/verb ambiguous words. University of Western Ontario, Department of Psychology, Research Bulletin No. 83, 1968.

UNDERWOOD, B. J. Stimulus selection in verbal learning. In C. N. Cofer and B. S. Musgrave (Eds.), Verbal behavior and learning. New York: McGfaw-Hill, 1963.

YNGVE, V. H. Implications of mechanical translation research. Proceedings of the American Philosophical Society, 1964, 108, $275-281$

NOTE

1. This research was supported in part by Grant OMHF 125 from the Ontario Mental Health Foundation. The authors wish to acknowledge the assistance of Dr. A. Paivio in the preparation of this manuscript. 